Supporting Information

\title{
Optimally Chiral Light: Upper Bound of Helicity Density of Structured Light for Chirality Detection of Matter at Nanoscale
}

\author{
Mina Hanifeh, Mohammad Albooyeh, and Filippo Capolino \\ Department of Electrical Engineering and Computer Science, University of California, Irvine, California 92697, USA.
}

Number of pages: 8

Number of figures: 2 


\section{A. Helicity of electromagnetic fields}

Linear and angular momenta of charged particles interacting with electromagnetic fields are conserved, accounting for the portion of these fiundamental quantities that may be transferred to electromagnetic fields; indeed these mechanical quantities are also defined for classical electromagnetic fields ${ }^{1}$. Helicity as one of the conserved quantities for classical electromagnetic fields has been defined based on mathematical concepts rather than mechanical ones, in terms of quantified knottedness or curlieness of a vector field ${ }^{2}$. In this regard, helicity density for time harmonic electromagnetic fields reads ${ }^{2-4}$ (when using the International System of Units)

$$
h=\frac{1}{4 c_{0}}\left(\epsilon_{0} \mathbf{F}^{*} \cdot \nabla \times \mathbf{F}+\mu_{0}^{-1} \mathbf{A}^{*} \cdot \nabla \times \mathbf{A}\right)
$$

where $\mathbf{A}$ and $\mathbf{F}$ are, respectively, the magnetic and electric vector potentials related to the electric $\mathbf{E}$ and magnetic $\mathbf{H}$ fields via $\mathbf{E}=\nabla \times \mathbf{F}$ and $\mathbf{H}=\mu_{0}^{-1} \nabla \times \mathbf{A}$, and $c_{0}=1 / \sqrt{\mu_{0} \epsilon_{0}}$ is the speed of light in vacuum. A monochromatic

field time dependence $e^{-i \omega t}$, where $\omega$ is the angular frequency, is implicitly assumed here and in the rest of the document.

Further investigation has clarified the physical significance of helicity, and it was shown that the mathematical integration of $h$ defined in Eq.(1) over the whole space is indeed related to the classical limit of the difference between the number of right-handed and left-handed photons in the field ${ }^{3}$. Helicity is also closely related to optical chirality, whose time-averaged expression is defined as ${ }^{5,6}$

$$
C=\frac{1}{4}\left(\epsilon_{0} \mathbf{E}^{*} \cdot \nabla \times \mathbf{E}+\mu_{0}^{-1} \mathbf{B}^{*} \cdot \nabla \times \mathbf{B}\right)
$$

which is a quantitative measure of handedness of electromagnetic fields to represent the strength of field for detection of chirality of matter ${ }^{5,7}$. Indeed, for time-harmonic electromagnetic fields, the time-averaged optical chirality and helicity are proportional: $C=\omega^{2} c_{0}^{-1} h$. In this manuscript, we adopt helicity as a measure to quantify fields' chirality.

\section{B. Properties of spin and angular momentum of optimally chiral fields}

We provide some properties of spin, angular momentum (AM) and momentum of a monochromatic (time-harmonic) optimally-chiral field. We recall that an optimally chiral field satisfies conditions (3) in the manuscript. The time dependence $e^{-i \omega t}$, where $\omega$ is the angular frequency, is implicitly assumed and SI units are employed. According to the Poynting Theorem ${ }^{1}$ in phasor terms, the power of an electromagnetic field generated by a current density $\mathbf{j}_{\text {elec }}$ in a volume $V$ satisfies

$$
-\frac{1}{2} \int_{V} \mathbf{j}_{\mathrm{elec}} \cdot \mathbf{E}^{*} d v=2 \omega i \int_{V}\left(u_{\mathrm{e}}-u_{\mathrm{m}}\right) d v+\int_{V} \nabla \cdot \mathbf{S}_{\mathrm{power}} d v
$$

Here, $u_{\mathrm{e}}=\mathbf{E} \cdot \mathbf{D}^{*} / 4$ and $u_{\mathrm{m}}=\mathbf{B} \cdot \mathbf{H}^{*} / 4$ are, respectively, the electric and magnetic energy densities, and $\mathbf{S}_{\text {power }}=\left(\mathbf{E} \times \mathbf{H}^{*}\right) / 2$ is the energy flux density of the field. The time-averaged physical values are obtained by taking the real part. Here, for simplicity we assume that electromagnetic fields are in vacuum. In phasor terms, density of momentum and $\mathrm{AM}$ of an electromagnetic field are, respectively, defined as $\boldsymbol{p}=\epsilon_{0}\left(\mathbf{E} \times \mathbf{B}^{*}\right) / 2$ and $\boldsymbol{j}=\mathbf{r} \times \boldsymbol{p}$, and satisfy conservation rules ${ }^{1,8}$. The total AM of the electromagnetic field in the volume $V$, given by $\mathcal{J}=\int_{V} j d v$, is decomposed into three parts ${ }^{9}$ :

$\mathcal{J}=\mathcal{L}+\mathcal{S}+\boldsymbol{\Omega}$ 
where $\mathcal{L}=\int_{V} \ell d v$ and $\mathcal{S}=\int_{V} s d v$ are, respectively, the total orbital AM (OAM) and the total spin AM (SAM) of the field. The densities of orbital $\ell$ and spin $s$ anular momenta of the field are obtained as ${ }^{4,9-12}$

$$
\begin{aligned}
& \boldsymbol{\ell}=\frac{\epsilon_{0}}{4} \mathbf{r} \times\left(\sum_{j=x, y, z} E_{j} \nabla A_{j}^{*}-\sum_{j=x, y, z} B_{j} \nabla F_{j}^{*}\right) \\
& \boldsymbol{s}=\frac{\epsilon_{0}}{4}\left(\mathbf{E} \times \mathbf{A}^{*}+\mathbf{F} \times \mathbf{B}^{*}\right),
\end{aligned}
$$

where $\mathbf{A}$ and $\mathbf{F}$ are auxiliary magnetic and electric vector potentials that give $\mathbf{E}=\nabla \times \mathbf{F}$ and $\mathbf{B}=\nabla \times \mathbf{A}$. Note that Coulomb gauge $\nabla \cdot \mathbf{A}=\mathbf{0}$ and $\nabla \cdot \mathbf{F}=\mathbf{0}$ is used ${ }^{13}$, and the Heaviside-Larmor symmetry in Maxwel's equation in free space is considered by employing both of electric and magnetic vector potentials $4,8,10,13$. Moreover, following the same method in Ref. ${ }^{9}$ for both auxiliary electric and magnetic vector potentials (only the part with the electric vector potential $\mathbf{F}$ is given in Ref. ${ }^{9}$ ), the third term in the right hand side of Eq. (4) in this Supplementary Information reads

$$
\boldsymbol{\Omega}=\frac{\epsilon_{0}}{4} \int_{\partial V}\left[\mathbf{B}\left(\mathbf{r} \times \mathbf{F}^{*}\right)-\mathbf{E}\left(\mathbf{r} \times \mathbf{A}^{*}\right)\right] \cdot \mathbf{d s} .
$$

In an optimally chiral field, the electric $\mathbf{E}$ and magnetic $\mathbf{H}$ fields are linked via the relation

$$
\mathbf{E}= \pm i \eta_{0} \mathbf{H}
$$

Therefore, the densities of linear momentum, orbital AM, and spin AM of an optimally chiral field are obtained as

$$
\begin{aligned}
\boldsymbol{p}^{ \pm} & =\frac{\mp i}{2 c_{0}^{2} \eta_{0}}\left(\mathbf{E} \times \mathbf{E}^{*}\right)=\frac{\mp i \eta_{0}}{2 c_{0}^{2}}\left(\mathbf{H} \times \mathbf{H}^{*}\right)=\frac{\mp i}{4 c_{0}^{2} \eta_{0}}\left(\mathbf{E} \times \mathbf{E}^{*}+\eta_{0}^{2} \mathbf{H} \times \mathbf{H}^{*}\right) \\
\boldsymbol{s}^{ \pm} & =-\frac{\epsilon_{0}}{4 i \omega}\left(\mathbf{E} \times \mathbf{E}^{*}+\eta_{0}^{2} \mathbf{H} \times \mathbf{H}^{*}\right) \\
\boldsymbol{\ell}^{ \pm} & =\frac{\epsilon_{0}}{4 i \omega} \mathbf{r} \times\left(\sum_{j=x, y, z} E_{j} \nabla E_{j}^{*}-\sum_{j=x, y, z} E_{j} \nabla E_{j}^{*}\right)=0 .
\end{aligned}
$$

In these equations the super script \pm refers to the \pm sign used in the relation $\mathbf{E}= \pm i \eta_{0} \mathbf{H}$. Note also that the integral in Eq.(6) vanishes, i.e., $\Omega=0$ for optimally chiral fields. Equation (8) shows that the orbital AM $\ell$ of an optimally chiral field vanishes and therefore, in an optimally chiral field, the total AM consists purely of the SAM. Moreover, the above relations show that the spin $\mathrm{AM} \boldsymbol{s}^{ \pm}$and the linear momentum $\boldsymbol{p}^{ \pm}$densities in an optimally chiral field are collinear.

\section{Poynting Theorem for fields in chiral media}

We discuss the power flow in chiral media using the Poynting theorem. Let us assume a lossy and dispersionless chiral medium occupies a volume $V$ enclosed by a surface $S$ and we assume there is no electromagnetic source inside it. According to Eq. (1) in this Supplementary Information, the total net time-average power flowing out of the surface $S$ reads

$$
\int_{S} \mathfrak{R}\left\{\mathbf{S}_{\text {power }}\right\} \cdot \mathbf{d s}=2 \omega \int_{V} \mathfrak{I}\left\{u_{\mathrm{e}}-u_{\mathrm{m}}\right\} d v
$$

where, $u_{\mathrm{e}}=\mathbf{E} \cdot \mathbf{D}^{*} / 4$ and $u_{\mathrm{m}}=\mathbf{B} \cdot \mathbf{H}^{*} / 4$. Substituting the constitutive relations $\mathbf{D}=\epsilon \mathbf{E}+i \kappa \sqrt{\mu_{0} \epsilon_{0}} \mathbf{H}$ and $\mathbf{B}=\mu \mathbf{H}-i \kappa \sqrt{\mu_{0} \epsilon_{0}} \mathbf{E}$ into the right hand side of Eq.(9), the integrand $\Im\left\{u_{\mathrm{e}}-u_{\mathrm{m}}\right\}$ reads 


$$
\mathfrak{I}\left\{u_{\mathrm{e}}-u_{\mathrm{m}}\right\}=-\frac{\mathfrak{I}\{\epsilon\}}{4}|\mathbf{E}|^{2}-\frac{\mathfrak{I}\{\mu\}}{4}|\mathbf{H}|^{2}-\frac{\sqrt{\mu_{0} \epsilon_{0}} \mathfrak{I}\{\kappa\}}{2} \mathfrak{J}\left\{\mathbf{E} \cdot \mathbf{H}^{*}\right\}
$$

Therefore the power conservation law is rewritten as

$$
-\int_{S} \mathfrak{R}\left\{\mathbf{S}_{\text {power }}\right\} \cdot \mathbf{d} \mathbf{s}=2 \omega \int_{V}\left(\frac{\mathfrak{I}\{\epsilon\}}{4}|\mathbf{E}|^{2}+\frac{\mathfrak{I}\{\mu\}}{4}|\mathbf{H}|^{2}+\frac{\sqrt{\mu_{0} \epsilon_{0}} \mathfrak{I}\{\kappa\}}{2} \mathfrak{J}\left\{\mathbf{E} \cdot \mathbf{H}^{*}\right\}\right) d v .
$$

This equation shows the net time-average power entering the volume $V$ (the left hand side) is equal to the the power dissipated inside such volume represented by the three terms on the right hand side: the first two are familiar terms, whereas the third one is proportional to the imaginary part of the chirality parameter $\kappa$; moreover, the sign of the dissipation of the chiral term with $\mathfrak{I}\{\kappa\}$ depends also on the sign of $\mathfrak{I}\left\{\mathbf{E} \cdot \mathbf{H}^{*}\right\}$. The fundamental constraints the constitutive parameters have to satisfy in a lossy medium are $\mathfrak{I}\{\epsilon\}>0, \quad \mathfrak{I}\{\mu\}>0 \quad$ and $(\mathfrak{I}\{\kappa\})^{2}<\mathfrak{I}\{\epsilon\} \mathfrak{I}\{\mu\} /\left(\epsilon_{0} \mu_{0}\right)$, as discussed in Ch. 2 of Ref. ${ }^{14}$. In a lossless medium, $\kappa$ is purely real.

D. Platform for chirality characterization of NPs based on two experiments with opposite helicity

We present in details the theoretical platform to characterize, i.e., to determine, the exact strength of optical chirality of an individual NP by utilizing the dissymmetry factor $g$, assuming the chiral NP interacts with an electromagnetic field not necessarily made of circularly polarized light. We consider monochromatic fields with time dependence $e^{-i \omega t}$ where $\omega$ is the angular frequency.

We consider a NP whose optical response is well approximated by the fundamental electric and magnetic dipole moments, and higher order multipoles are considered negligible, which is a judicious and common assumption for optically small NPs. Moreover, we assume that the NP is isotropic, reciprocal, and is surrounded by an isotropic nondispersive medium and hence induced electric $\mathbf{p}$ and magnetic $\mathbf{m}$ dipole moments are linearly related to local electric $\mathbf{E}$ and magnetic $\mathbf{H}$ fields via polarizabilities through relations ${ }^{15}$

$\mathbf{p}=\alpha_{\mathrm{ee}} \mathbf{E}+\alpha_{\mathrm{em}} \mathbf{H}, \quad \mathbf{m}=-\mu_{0}^{-1} \alpha_{\mathrm{em}} \mathbf{E}+\alpha_{\mathrm{mm}} \mathbf{H}$

Here $\alpha_{\mathrm{ee}}$ and $\alpha_{\mathrm{mm}}$ are the electric and magnetic polarizabilities of the NP, respectively. Moreover, the magnetoelectric polarizability $\alpha_{\mathrm{em}}$ describes the NP's optical chirality, and determining this quantity is the goal of chirality characterization of the NP. The magnetic dipole moment is defined as $\mathbf{m}=\frac{1}{2} \int_{\mathrm{V}} \mathbf{r} \times \mathbf{J}(\mathbf{r}) \mathrm{d} \boldsymbol{u}$ where $\mathbf{J}(\mathbf{r})$ is displacement current density in the NP. Employing Eq. (12) in the Poynting theorem ${ }^{1}$, the time-averaged extinction power $P_{\text {ext }}$, which is a convenient representative of the NP's interaction with light, is obtained as ${ }^{\mathbf{1 6}}$

$$
P_{\mathrm{ext}}=2 \epsilon_{0}^{-1} \omega \mathfrak{I}\left\{\alpha_{\mathrm{ee}}\right\} u_{\mathrm{e}}+2 \omega \mathfrak{I}\left\{\alpha_{\mathrm{mm}}\right\} u_{\mathrm{m}}-2 c_{0} \omega^{2} \mathfrak{R}\left\{\alpha_{\mathrm{em}}\right\} h
$$

Here, $u_{\mathrm{e}}=\epsilon_{0}|\mathbf{E}|^{2} / 4$ and $u_{\mathrm{m}}=\mu_{0}|\mathbf{H}|^{2} / 4$ are, respectively, the time-averaged electric and magnetic energy densities of the local field. Moreover, the time-averaged helicity density of the local field acting on the NP reads ${ }^{2-}$ 4,8,17

$$
h=\frac{\mathfrak{I}\left\{\mathbf{E} \cdot \mathbf{H}^{*}\right\}}{2 \omega c_{0}},
$$


where $\mathfrak{I}\{\}$ denotes for the imaginary part of a complex value. Note that when helicity of the NP's illuminating field is different from zero, the extinction power depends on the sign and value of the chirality parameter $\mathfrak{R}\left\{\alpha_{\mathrm{em}}\right\}$; viceversa, when $h=0$, the extinction power is totally independent of $\Re\left\{\alpha_{\mathrm{em}}\right\}$.

To reveal the electromagnetic polarizability $\alpha_{\mathrm{em}}$ of a NP, we follow the scheme used in circular dichroism, adapted to the NP case. Therefore, we consider the interaction of the NP with two distinct excitations and denote the measured extinctions in the two "experiments" by superscripts " + " and " -", respectively (the + and - signs are chosen since the two excitations possess positive and negative helicity densities). The difference between the extinction powers in the two interactions $\Delta P_{\mathrm{ext}}=P_{\mathrm{ext}}^{+}-P_{\mathrm{ext}}^{-}$, which we call differential extinction, reads

$\Delta P_{\mathrm{ext}}=2 \omega \epsilon_{0}^{-1} \mathfrak{J}\left\{\alpha_{\mathrm{ee}}\right\} \Delta u_{\mathrm{e}}+2 \omega \mathfrak{I}\left\{\alpha_{\mathrm{mm}}\right\} \Delta u_{\mathrm{m}}-2 c_{0} \omega^{2} \mathfrak{R}\left\{\alpha_{\mathrm{em}}\right\} \Delta h$,

where $\Delta h=h^{+}-h^{-}, \Delta u_{\mathrm{e}}=u_{\mathrm{e}}^{+}-u_{\mathrm{e}}^{-}$, and $\Delta u_{\mathrm{m}}=u_{\mathrm{m}}^{+}-u_{\mathrm{m}}^{-}$are, respectively, the difference between helicity, electric energy, and magnetic energy densities in two experiments. In the chirality detection algorithm described in the main body of he paper, it is convenient to eliminate the contribution of the electric and magnetic polarizabilities, i.e., the first two terms of Equation (15). This is achived when

$$
\begin{aligned}
& \Delta u_{\mathrm{m}}=u_{\mathrm{m}}^{+}-u_{\mathrm{m}}^{-}=0, \\
& \Delta u_{\mathrm{e}}=u_{\mathrm{e}}^{+}-u_{\mathrm{e}}^{-}=0 .
\end{aligned}
$$

Therefore, when the electric and magnetic local-field energy densities in the two + and - experiments are equal, the differential extinction $\Delta P_{\text {ext }}$ reads

$$
\Delta P_{\mathrm{ext}}=-2 c_{0} \omega^{2} \Re\left\{\alpha_{\mathrm{em}}\right\} \Delta h
$$

This equation suggests that higher values of $\Delta h$ correspond to a better capability ofdetection of smaller values of chirality, i.e., smaller values of $\mathfrak{R}\left\{\alpha_{\mathrm{em}}\right\}$. Though the differential extinction (17) does not include anymore the contributions of the electric and magnetic polarizabilities thanks to conditions (16), yet it contains the fields' property $\Delta h$ besides the sought NP's optical chirality $\mathfrak{R}\left\{\alpha_{\mathrm{em}}\right\}$. The next important step is obtained by considering the dissymmetry factor $g$ defined as 5,18

$$
g=\frac{\Delta P_{\text {ext }}}{\bar{P}_{\text {ext }}}
$$

to eliminate the field properties $\Delta h$ from Eq. (17), where $\bar{P}_{\text {ext }}$ is the arithmetic average of the extinction powers. After imposing conditions (16) to excitations, the dissymmetry factor $g$ in Eq. (18) reads

$$
g=\frac{-2 c_{0} \omega^{2} \Re\left\{\alpha_{\mathrm{em}}\right\} \Delta h}{2 \omega \epsilon_{0}^{-1} \mathfrak{J}\left\{\alpha_{\mathrm{ee}}\right\} u_{\mathrm{e}}+2 \omega \mathfrak{I}\left\{\alpha_{\mathrm{mm}}\right\} u_{\mathrm{m}}+2 c_{0} \omega^{2} \mathfrak{R}\left\{\alpha_{\mathrm{em}}\right\} \bar{h}},
$$

where $u_{\mathrm{m}}=u_{\mathrm{m}}^{+}=u_{\mathrm{m}}^{-}, u_{\mathrm{e}}=u_{\mathrm{e}}^{+}=u_{\mathrm{e}}^{-}$, and $\bar{h}=\left(h^{+}+h^{-}\right) / 2$. Yet, more restrictions on fields are needed to remove the field properties from Eq. (19), to make $g$ directly related to the NP chirality $\mathfrak{R}\left\{\alpha_{\mathrm{em}}\right\}$, independelty of the value of the field chirality. Therefore we introduce the general condition, applicable to any kind of structured light used in the two experiments,

$$
h^{+}=-h^{-}=h,
$$


which means that fields in the two experiments possess helicity densities with equal amplitudes and opposite signs. Hence, the last term in denominator of Eq. (19) is eliminated and this equation reduces to

$$
g=\frac{-2 c_{0} \omega \Re\left\{\alpha_{\mathrm{em}}\right\} h}{\epsilon_{0}^{-1} \mathfrak{J}\left\{\alpha_{\mathrm{ee}}\right\} u_{\mathrm{e}}+\mathfrak{I}\left\{\alpha_{\mathrm{mm}}\right\} u_{\mathrm{m}}} .
$$

We also propose to employ two optimally chiral fields with time-averaged energy and helicity densities satisfying the relation $|h|=2 u_{\mathrm{e}} / \omega=2 u_{\mathrm{m}} / \omega=u / \omega$, where $u=u_{\mathrm{e}}+u_{\mathrm{m}}$ is the total time-averaged energy density of the field, to better characterize the NP's optical chirality via the dissymmetry factor $g$. (Optimally chiral fields have $u_{\mathrm{e}}=u_{\mathrm{m}}$.) Therefore, the two fields used in the two experiments shall satisfy the conditions

$\mathbf{E}^{ \pm}= \pm i \eta_{0} \mathbf{H}^{ \pm}$

where $\eta_{0}$ is the intrinsic wave impedance of vacuum (we recall that for simplicity we assume the NP to be in vacuum/free space), which means that they are both optimally chiral fields. Note that the + and - signs in Eq. (22) correspond, respectively, to fields with positive and negative helicity densities, since

$$
h^{ \pm}=\frac{\mathfrak{I}\left\{\mathbf{E}^{ \pm} \cdot\left(\mathbf{H}^{ \pm}\right)^{*}\right\}}{2 \omega c_{0}}= \pm \frac{\left|\mathbf{E}^{ \pm}\right|^{2}}{2 \omega \eta_{0} c_{0}}= \pm \frac{u^{ \pm}}{\omega} .
$$

The introduction of optimally chiral fields in Eq. (21) simplifies the dissymmetry factor $g$ to

$$
g=\frac{-4 c_{0} \mathfrak{R}\left\{\alpha_{\mathrm{em}}\right\}}{\epsilon_{0}^{-1} \mathfrak{J}\left\{\alpha_{\mathrm{ee}}\right\}+\mathfrak{I}\left\{\alpha_{\mathrm{mm}}\right\}},
$$

which describes the NP's chirality in terms of its electric and magnetic polarizabilities, and it is independent of the field's property. This result is general and it applies to any striuctured light that satisfies the optimal chirality condition. Moreover, for NPs with weak magnetic polarizability, i.e., when $\left.\epsilon_{0}^{-1} \Im\left\{\alpha_{\mathrm{ee}}\right\}>>\Im \alpha_{\mathrm{mm}}\right\}$, which is a rather common assumption, the dissymmetry factor $g$ reduces to

$$
g=-4 \eta_{0}^{-1} \mathfrak{R}\left\{\alpha_{\mathrm{em}}\right\} / \mathfrak{I}\left\{\alpha_{\mathrm{ee}}\right\}
$$

In summary, when using optimally chiral fields, the $g$ factor describes the ratio between the magnetoelectric and electric polarizabilities, i.e., it characterizes the NP's chirality relative to the NP's electric polarizability $\alpha_{\mathrm{ee}}$. Indeed, note that both these two quantities scale with the NP dimension, hence the $g$ factor mainly describes the material composing the chiral nanosphere.

\section{E. Constitutive relations and chirality parameter of Poly-L-glutamic Acid (PGA)}

The real and imaginary parts of the chirality parameter $\kappa$ used in the calculation of the polarizabilities of Poly-LGlutamatic acid spherical particles are obtained from Appendix B of Ref. ${ }^{19}$. The rotatory strength and linewidth of the absorption bands in the Drude models assumed for $n=\left(n_{+}+n_{-}\right) / 2$ and $\Delta n=n_{+}-n_{-}$are calculated to fit the experimental data of $n_{-}$and $n_{+}$in Ref. ${ }^{20}$. Note that $n_{-}$and $n_{+}$are the two refractive indices of circularly polarized (CP) light in a PGA medium corresponding to right and left handed circular polarizations

The chirality parameter $\kappa$ in the constitutive relations $\mathbf{D}=\epsilon \mathbf{E}+i \kappa \sqrt{\mu_{0} \epsilon_{0}} \mathbf{H}$ and $\mathbf{B}=\mu \mathbf{H}-i \kappa \sqrt{\mu_{0} \epsilon_{0}} \mathbf{E}$ describes the bulk material made of PGA ${ }^{\mathbf{1 4}}$. The chirality parameter $\kappa$ and the refractive index $\sqrt{\epsilon \mu / \epsilon_{0} \mu_{0}}$ are related to the CP refractive indeces as ${ }^{14}$ 


$$
\kappa=\frac{n_{+}-n_{-}}{2} \quad \sqrt{\frac{\epsilon \mu}{\epsilon_{0} \mu_{0}}}=\frac{n_{+}+n_{-}}{2} .
$$

The electric, magnetic, and electromagnetic polarizabilities $\alpha_{\mathrm{ee}}, \alpha_{\mathrm{mm}}$, and $\alpha_{\mathrm{em}}$ for a spherical chiral nanoparticle made of PGA are calculated using the formulas for the $b_{1}, a_{1}$, and $c_{1}$ coefficients at page 15 in Chapter III of Bohren's $\mathrm{PhD}$ Thesis ${ }^{19}$, as

$$
\alpha_{\mathrm{ee}}=-\frac{6 \pi i}{k^{3}} \epsilon_{0} b_{1}, \quad \alpha_{\mathrm{mm}}=-\frac{6 \pi i}{k^{3}} a_{1}, \quad \text { and } \quad \alpha_{\mathrm{em}}=-\frac{6 \pi i}{k^{3}} c_{0} c_{1}
$$

where $c_{0}$ is the speed of light in vacuum and $k=\omega / c_{0}$. (Note that contrarily to a common notation, following Bohern's Thesis one has $\alpha_{\mathrm{ee}} \propto b_{1}$ and not $\alpha_{\mathrm{ee}} \propto a_{1}$, i.e., the role of the coefficients is inverted with respect to what used in several other works.) As discussed in Ref. ${ }^{19}$, we assume that $\mu \approx \mu_{0}$ in the evaluation of the polarizabilities, since dissipation losses due to the magnetic permeability are smaller than the other dissipation losses, hence it is assumed that $\sqrt{\epsilon / \epsilon_{0}} \approx\left(n_{+}+n_{-}\right) / 2$. Alternatively, the coefficients $a_{1}, b_{1}$, and $c_{1}$ could also be obtained using formulas at page 18 of Bohren's PhD Thesis ${ }^{19}$ based on Riccati-Bessel functions.

The imaginary and real parts of the chirality parameter $\kappa$ for PGA are depicted in Figure S1, and they are used in the main body of the paper to calculate the electromagnetic polarizability $\alpha_{\mathrm{em}}$ of a spherical nano sample of PGA.

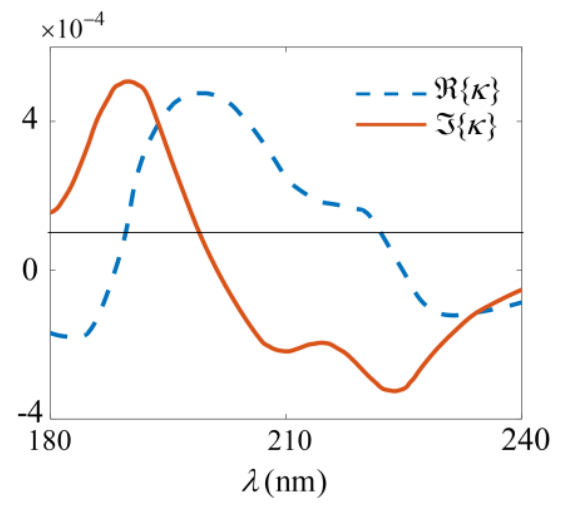

Figure S1. Real and imaginary parts of the chirality parameter $\kappa$ of poly-L-glutamic acid (PGA). This data is used in the evaluation of the electromagnetic polarizability $\alpha_{\mathrm{em}}$ of a nanosphere made of such material in the main body of the paper.

\section{F. Helicity density of a Gaussian beam with circular polarization}

Here, we evaluate helicity density of a Gaussian beam (GB) with circular polarization propagating along the $+z$ direction. The electric field vector in a GB with circular polarization, under paraxial approximation, reads ${ }^{\mathbf{2 1}}$

$$
\mathbf{E}=\sqrt{\frac{2}{\pi}} \frac{V_{G}}{w} e^{-(\rho / w)^{2}} e^{i k \rho^{2} /(2 R)} e^{-i \tan ^{-1}\left(z / z_{\mathrm{R}}\right)} e^{i k z}(\hat{\mathbf{x}} \pm i \hat{\mathbf{y}})
$$

where the $+/$ - sign represents left/ right -handed circular polarizations (Ch. 7.2 in Ref. ${ }^{1}$ ), and $\hat{\mathbf{x}}$ and $\hat{\mathbf{y}}$ are the unit vectors of the Cartesian coordinate system. Here, $z_{\mathrm{R}}=\pi w_{0}^{2} / \lambda, w=w_{0} \sqrt{1+\left(z / z_{\mathrm{R}}\right)^{2}}$, and $R=z\left[1+\left(z_{R} / z\right)^{2}\right]$ with $w_{0}$ and $\lambda$ being, respectively, the beam parameter and the excitation wavelength, respectively. The beam parameter $w_{0}$ represents half of the beam waist when the beam is not tightly focused ${ }^{22}$. Moreover, $\rho$ and $V_{\mathrm{G}}$ are, respectively, 
the radial distance from the beam axis (the $z$-axis of the Cartesian coordinate system) and the complex amplitude of the beam (with the unit of Volt). In calculating helicity, the magnetic field component of a GB is obtained from the Maxwell equation $\mathbf{H}=\left(i \omega \mu_{0}\right)^{-1} \nabla \times \mathbf{E}$.

In Figure S2 (a), we illustrate the helicity density of a circularly polarized GB with total power of $1 \mathrm{~mW}$ and beam parameter $w_{0}=\lambda=210 \mathrm{~nm}$ evaluated at the $z=0$ plane. This figure shows that the maximum value of the helicity density occurs on the beam's optical axis $\rho=0$. In Figure S2 (b), the helicity density of the described GB at $z=\rho=0$ is depicted for various wavelengths.
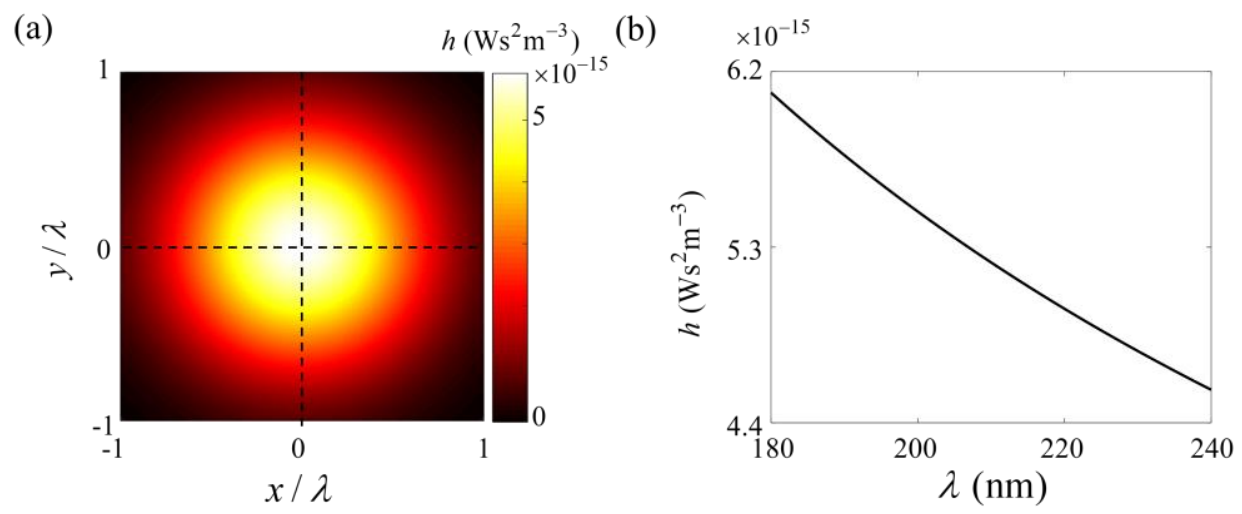

Figure S2. Helicity density of a Gaussian beam with circular polarization and beam parameter $w_{0}=\lambda$, propagating along the $z$ direction, (a) in the $x y$ plane $(z=0)$ at the operational wavelength $\lambda=210 \mathrm{~nm}$, and (b) on the optical axis at $z=\rho=0$ versus wavelength ( still $w_{0}=\lambda$ ). The total power of the beam is $1 \mathrm{~mW}$.

\section{References}

(1) Jackson, J. D. Classical Electrodynamics, 3rd Edition; John Wiley \& Sons, New York, 1998.

(2) Ranada, A. F. On the Magnetic Helicity. Eur. J. Phys. 1992, 13 (2), 70. https://doi.org/10.1088/01430807/13/2/003.

(3) Trueba, J. L.; Rañada, A. F. The Electromagnetic Helicity. Eur. J. Phys. 1996, 17 (3), 141. https://doi.org/10.1088/0143-0807/17/3/008.

(4) Cameron, R. P.; Barnett, S. M.; Yao, A. M. Optical Helicity, Optical Spin and Related Quantities in Electromagnetic Theory. New J. Phys. 2012, 14 (5), 053050. https://doi.org/10.1088/13672630/14/5/053050.

(5) Tang, Y.; Cohen, A. E. Optical Chirality and Its Interaction with Matter. Phys. Rev. Lett. 2010, 104 (16), 163901. https://doi.org/10.1103/PhysRevLett.104.163901.

(6) Vázquez-Lozano, J. E.; Martínez, A. Optical Chirality in Dispersive and Lossy Media. Phys. Rev. Lett. 2018, 121 (4), 043901. https://doi.org/10.1103/PhysRevLett.121.043901.

(7) Tang, Y.; Cohen, A. E. Enhanced Enantioselectivity in Excitation of Chiral Molecules by Superchiral Light. Science 2011, 332 (6027), 333-336. https://doi.org/10.1126/science.1202817.

(8) Bliokh, K. Y.; Bekshaev, A. Y.; Nori, F. Dual Electromagnetism: Helicity, Spin, Momentum and Angular Momentum. New J. Phys. 2013, 15 (3), 033026. https://doi.org/10.1088/1367-2630/15/3/033026.

(9) Stewart, A. M. Angular Momentum of the Electromagnetic Field: The Plane Wave Paradox Resolved. European Journal of Physics 2005, 26 (4), 635-641.

(10) Barnett, S. M. Rotation of Electromagnetic Fields and the Nature of Optical Angular Momentum. Journal of Modern Optics 2010, 57 (14-15), 1339-1343.

(11) Barnett, S. M.; Allen, L. Orbital Angular Momentum and Nonparaxial Light Beams. Optics Communications 1994, 110 (5), 670-678. https://doi.org/10.1016/0030-4018(94)90269-0. 
(12) Ohanian, H. C. What Is Spin? American Journal of Physics 1986, 54 (6), 500-505.

(13) Barnett, S. M.; Cameron, R. P.; Yao, A. M. Duplex Symmetry and Its Relation to the Conservation of Optical Helicity. Phys. Rev. A 2012, 86 (1), 013845. https://doi.org/10.1103/PhysRevA.86.013845.

(14) Lindell, I.; Sihvola, A.; Tretyakov, S.; Viitanen, A. Electromagnetic Waves in Chiral and Bi-Isotropic Media; Artech House, 1994.

(15) Serdiukov, A.; Semchenko, I.; Tretyakov, S.; Sihvola, A. Electromagnetics of Bi-Anisotropic Materials Theory and Application; Gordon and Breach Science Publishers, 2001; Vol. 11.

(16) Hanifeh, M.; Albooyeh, M.; Capolino, F. Empowering Structured Light to Enhance Chirality Detection and Characterization at Nanoscale. In Complex Light and Optical Forces XIII; International Society for Optics and Photonics, 2019; Vol. 10935, p 1093504. https://doi.org/10.1117/12.2509042.

(17) Fernandez-Corbaton, I.; Zambrana-Puyalto, X.; Tischler, N.; Vidal, X.; Juan, M. L.; Molina-Terriza, G. Electromagnetic Duality Symmetry and Helicity Conservation for the Macroscopic Maxwell's Equations. Phys. Rev. Lett. 2013, 111 (6), 060401.

(18) Kuhn, W. Optical Rotatory Power. Annual Review of Physical Chemistry 1958, 9 (1), 417-438.

(19) Bohren, C. F. Light Scattering by Optically Active Particles. Thesis, The University of Arizona, United States of America, 1975.

(20) Urry, D. W.; Krivacic, J. Differential Scatter of Left and Right Circularly Polarized Light by Optically Active Particulate Systems. PNAS 1970, 65 (4), 845-852.

(21) Veysi, M.; Guclu, C.; Capolino, F. Vortex Beams with Strong Longitudinally Polarized Magnetic Field and Their Generation by Using Metasurfaces. J. Opt. Soc. Am. B, JOSAB 2015, 32 (2), 345-354.

(22) Veysi, M.; Guclu, C.; Capolino, F. Focused Azimuthally Polarized Vector Beam and Spatial Magnetic Resolution below the Diffraction Limit. J. Opt. Soc. Am. B, JOSAB 2016, 33 (11), 2265-2277. 